15

\title{
Scintillations effects on satellite to Earth links for telecommunication and navigation purposes
}

\author{
YANNICK BÉNIGUEL $\left({ }^{1}\right)$, BIAGIO FORTE $\left({ }^{2}\right)$, SANDRO M. RADICELLA $\left({ }^{2}\right)$, \\ HAL J. STRANGEWAYS $\left({ }^{3}\right)$, VADIM E. GHERM $\left({ }^{4}\right)$ and NIKOLAY N. ZERNOV $\left(^{4}\right)$ \\ ( $\left.{ }^{1}\right)$ IEEA, Courbevoie, France \\ ( $\left.{ }^{2}\right)$ The Abdus Salam International Centre for Theoretical Physics (ICTP), \\ Aeronomy and Radiopropagation Laboratory, Trieste, Italy \\ $\left.{ }^{3}\right)$ University of Leeds, U.K. \\ $\left({ }^{4}\right)$ University of St. Petersburg, Russia
}

Radio wave scintillations are rapid fluctuations in both amplitude and phase of signals propagating through the atmosphere. GPS signals can be affected by these disturbances which can lead to a complete loss of lock when the electron density strongly fluctuates around the background ionization level at small spatial scales. This paper will present recent improvements to the theoretical Global Ionospheric Scintillation Model (GISM), particularly tailored for satellite based navigation systems such GPS coupled with Satellite Based Augmentation System (SBAS). This model has been improved in order to take into account GPS constellation, signals, and receiver response to ionospheric scintillation environments. A new modelling technique, able to describe the scintillation derived modifications of transionospheric propagating fields is shown. Results from GPS derived experimental measurements performed at high and low magnetic latitudes will show preliminary assessments of the scintillation impact on real receivers and system operations. Nevertheless, comparisons between theoretical scintillation models, such as WBMOD and GISM, with GPS derived experimental data will be shown.

\subsection{INTRODUCTION}

This paper presents a review of the scintillation problem on satellite to ground links. These scintillations result from propagation through the ionosphere inside which inhomogeneities have developed and create random variations of the medium index. These inhomogeneities create a number of modifications on the signals, among them phase and intensity fluctuations, fluctuations of the arrival angle, dispersivity, Doppler, etc. The ionosphere scintillations are especially important at equatorial and auroral regions. Equatorial scintillations appear after sunset and may last a few hours. They are related in particular to solar activity and the season.

Scintillation models usually only consider random fluctuations. The traveling disturbances which act as long term fluctuations with respect to the time scales considered do not contribute to the scintillations. Two models are presented in this paper: the first named GISM is based on a solution of the parabolic equation while the second uses a hybrid method. 
Yannick Béniguel, Biagio Forte, Sandro M. Radicella, Hal J. Strangeways, Vadim E. Gherm and Nikolay N. Zernov

GISM model developed at IEEA uses the Multiple Phase Screen technique (MPS). It consists in a resolution of the Parabolic Equation (PE) for a medium divided into successive layers, each of them acting as a phase screen. It provides the statistical characteristics of the transmitted signals, in particular the scintillation index, the fade durations and the cumulative probability of the signal consequently allowing determination of the margins to be included in a budget link. Maps of the scintillation index $S 4$ and of the phase standard deviation may also be obtained.

Within the scope of the activities of COST 271 relevant to the problem of transionospheric propagation another model for scintillation on transionospheric links (such as employed for satellite navigation) has been developed in co-operation between the University of St. Petersburg, St. Petersburg, Russia and the School of Electronic and Electrical Engineering, the University of Leeds, Leeds, U.K. The Abdus Salam ICTP, Trieste, Italy also collaborated with both the teams providing the experimental data on scintillation, ideas for proper processing of the scintillation data and necessary expertise and data on the ionosphere modeling.

The developed technique is based on a hybrid method and was first presented at the 27th General Assembly of URSI (Gherm et al., 2002b). It is an extension of the technique initially suggested in (Gherm et al., 2000). The extended method is a combination of the complex phase method and the technique of a random screen. For decades the theory of random phase screen was widely employed for interpretation of scintillation data. However, it is always the case that the parameters of the effective phase screen are chosen to better fit a given set of experimental data. The disadvantage of such a treatment is that the same parameters of the random screen cannot then be utilized for prediction of scintillation in different conditions of propagation. By contrast in the new hybrid method, the parameters of the random screen, appropriately introduced below the ionosphere, are not chosen empirically but are the results of a rigorous solution to the problem of propagation inside the ionosphere layer.

Detailed investigations in the scope of the complex phase method showed that, for the points of observation lying inside the ionosphere layer, fluctuations of the amplitude of a field at frequencies of the order of $1 \mathrm{GHz}$ and higher are always well within the range of validity of the complex phase method. This is true even in the case of very large relative electron density fluctuations (up to 100\%) and high values of the Total Electron Content (TEC). For smaller relative fluctuations and values of TEC, this is also true for lower frequencies. This means that propagation in the ionosphere layer for the frequencies mentioned may be rigorously described in the scope of the complex phase method. In turn, this means that at the specified frequencies, the regime of strong scintillation is not normally found inside the ionosphere layer, but may instead be found in the region below where the fields propagate down to the Earth's surface. This then permits the complex phase method to be used to properly introduce the random screen below the ionosphere, and then to employ the rigorous relationships of the random screen theory to correctly convey the field down to the surface of the Earth. While propagating the fields between the bottom of the ionosphere and the Earth's surface, the regime of strong scintillation may well be found.

Comparisons of models and measurements are presented.

The measurement techniques and detrending algorithms, the receiver transfer function and characterization and the effects of scintillations on positioning errors are also addressed in this paper.

\subsection{DEPENDENCY OF INHOMOGENEITIES ON LATITUDE}

Scintillations are most severe and prevalent in and north of the auroral zone and near the geomagnetic equator (Aarons, 1982). The equatorial region extends approximately from $-20^{\circ}$ to $20^{\circ}$ and auroral regions from $55^{\circ}$ to $90^{\circ}$. These boundaries change with the time of the day, the season of the year, the sunspot number and the magnetic activity.

Equatorial scintillations - Scintillation is predominantly a nighttime phenomenon in the equatorial region occurring for more than 40\% of the year during the 20:00-02:00 local time period (Basu et al., 
1986). It also shows a strong seasonal dependence with a pronounced minimum at the southern solstice and relatively high scintillation activity at the northern solstice. Equatorial scintillations also show a tendency to occur more often during years in which the sunspot number is high. The rms amplitude of electron density irregularities is equal to $20 \%$ in the most severe cases.

Two regimes may be identified. For values of the scintillation index ( $S 4$ ) below approximately 0.5 , the rms value of phase and intensity fluctuations seems to be linearly correlated and approximately equal. For greater values of $S 4$, there is no obvious correlation and measured values are greater for intensity than for phase (Doherty et al., 2000).

If we consider the case of GPS $L 1$ scintillations, the typical value of $S 4$ at equatorial regions is 0.3 . Its occurrence is related to the season and the solar activity. It may reach a value of 0.5 with an occurrence $10 \%$ below and a value of 0.8 or even 1 in a few cases.

High latitude scintillations - Contrary to equatorial fluctuations, according to measurements performed in Alaska, polar fluctuations exhibit more phase than intensity fluctuations. The scintillation index is usually quite low. It seldom exceeds 0.2 and the same for the probability of occurrence which is very low in summer and in any case below the values obtained at equatorial regions.

Results of measurements recently performed in Norway, seem to confirm the low values of $S 4$. The phase fluctuations are correlated to amplitude fluctuations and the $\sigma_{\phi}$ values obtained are also quite low.

\subsection{GISM Propagation Model}

\subsubsection{Medium modeling}

The electronic density inside the medium is calculated by the NeQuick model developed by the Universities of Graz and Trieste. Inputs of this model are the solar flux number, the year, the day of the year and the local time. It provides the electronic density average value for any point in the ionosphere (latitude, longitude, altitude). The NeQuick model is used as a subroutine in the GIM model.

\subsubsection{Algorithm}

Propagation errors are of two kinds: mean errors; scintillations and more generally higher order moments of the signal.

Mean errors are obtained solving the ray equation. The ray differential equation is solved by the Runge Kutta algorithm. The line of sight is defined taking the electron density gradient at each point along the ray into account. This is done with respect to the three axes in a geodesic referential system. This is an iterative algorithm. It is stopped when the ray crosses the plane perpendicular to the line of sight and containing the source point. The mean errors: range, angular and Faraday rotation are subsequently determined.

The calculation of fluctuations is a $2 \mathrm{D}$ calculation. The first dimension $z$ is the line of sight previously determined. The second dimension $x$ is perpendicular to this. Since the problem is solved as a two dimensional one, the medium of propagation is considered to be homogeneous in the third direction, which is perpendicular to the first two directions. The propagation of the monochromatic component of the field $E$ in the random medium is described by Helmholtz equation

$$
\nabla^{2} E+k^{2}(z)[1+\varepsilon(x, z)] E=0
$$

where $k^{2}(z)=\omega^{2} \varepsilon_{0}(z) / c^{2}$ is the local wave number, $\varepsilon_{0}(z)$ is the background dielectric permittivity along the main propagation axis $z, \varepsilon(x, z)$ is the random field of the relative dielectric permittivity. 
Introducing the complex amplitude $U(x, z)$ of the stochastic field

$$
E(x, z)=U(x, z) \exp \left(i \int_{0}^{z} k(z) d z\right)
$$

and assuming that the variation of the complex amplitude is mainly in the direction perpendicular to the main propagation axis (parabolic approximation), the stochastical parabolic equation for the complex amplitude can be written in the form

$$
2 i k \frac{\partial U}{\partial z}+\nabla_{t}^{2} U+k^{2} \varepsilon U=0
$$

where $\nabla_{t}^{2}$ is the transversal Laplacian.

To solve eq. (15.3), the medium is divided into a series of successive layers perpendicular to the main propagation axis, each one characterized by local homogeneous statistical properties. The solution is then obtained by iterating successively scattering and propagation calculations as detailed hereafter.

The parabolic wave eq. (15.3) is split into two equations. The first describes the phase change due to the presence of random fluctuations $\varepsilon(x, z)$

$$
2 i k \frac{\partial U}{\partial z}+k^{2} \varepsilon U=0
$$

and the second equation describes propagation in the space without fluctuations

$$
2 i k \frac{\partial U}{\partial z}+\nabla_{t}^{2} U=0
$$

Introducing the small step $\Delta z$ along the main propagation axis, the solution of the successive couple of eqs. ((15.4) and (15.5)) can be obtained in the form

$$
U(x, z+\Delta z)=\left(\frac{k}{2 i \pi \Delta z}\right)^{1 / 2} \int U\left(x^{\prime}, z\right) \exp \left(\frac{i k \Delta z}{2} \varepsilon\left(x^{\prime}, z\right)\right) \exp \left(\frac{i k\left(x-x^{\prime}\right)^{2}}{2 \Delta z}\right) d x^{\prime}
$$

In eq. (15.6) the first exponent under the integral sign arises from the solution to eq. (15.4), and the second exponent together with the factor before the integral sign is the Green's function for eq. (15.5). In practice the convolution integral (15.6) is calculated by means of fast Fourier transformation. Applying eq. (15.6) to each layer, the solution to the parabolic eq. (15.3) is obtained. This technique is referred in the literature as multiple phase screen technique.

\subsubsection{Random medium synthesis}

The spectral density of the phase at the output of the medium is written as the product of the Fourier transform of a centered gaussian random variable by the square root of the spectral density of the signal that we want to synthesize. The resulting random variable meets the required conditions. The corresponding signal is obtained taking the inverse Fourier transform of this product. 
15.3.2.2. Results obtained (figs. 15.1 and 15.2)
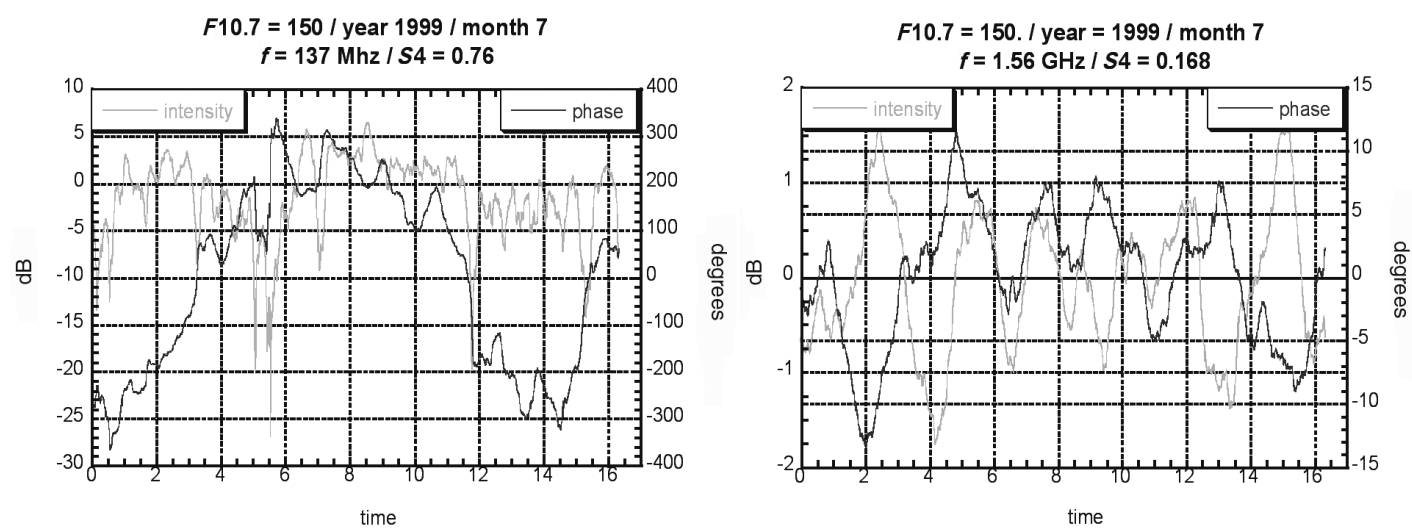

Fig. 15.1. Phase and intensity fluctuations: VHF- and $L$-band links.
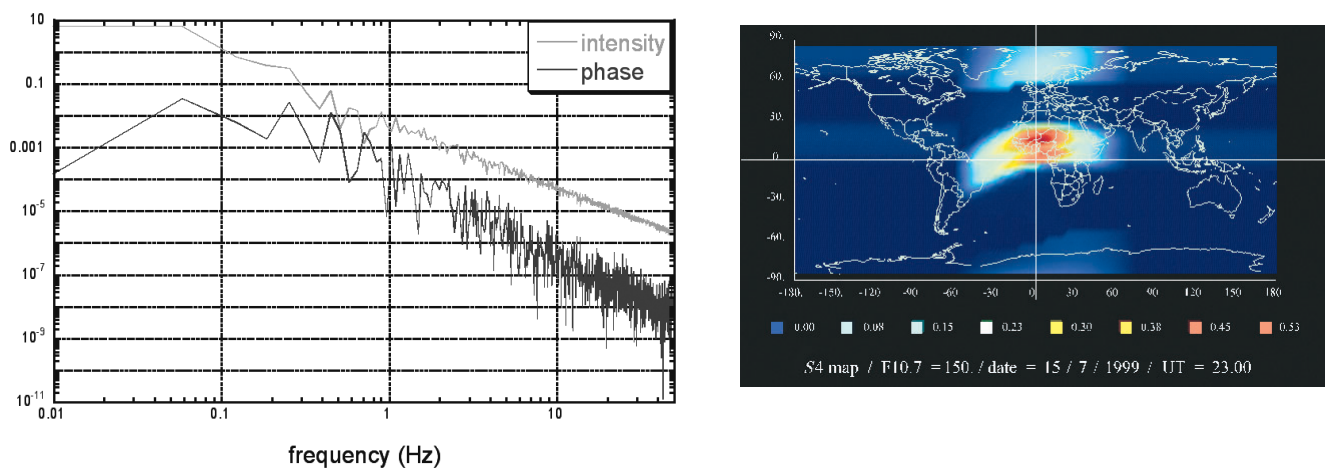

Fig. 15.2. The spectrum of transmitted signals and a global map.

\subsection{Hybrid MOdel}

The procedure includes generating a $2 \mathrm{D}$ random screen below the ionosphere by means of the complex phase method. Its random 2D spatial spectrum is then transferred down to the Earth's surface employing the propagator written in terms of the Fourier variable conjugated to the transversal variables in the following form:

$$
\widetilde{E}(z, \kappa, t)=e^{i t k} \widetilde{E}(z, \kappa, t) \exp \left(-\frac{i \kappa^{2} z}{2 k}\right) .
$$

Here $\tilde{E}(0, \kappa, t)$ is the transversal spectrum of a random field generated on the random screen, and $\tilde{E}(z, \kappa, t)$ is a random transversal spectrum of the field on the Earth's surface. Form (15.1) of the prop- 
agator follows from the theory of the random screen. Performing the inverse Fourier transformation yields a 2D random distribution of the field on the surface of the Earth. Finally, when the drift («frozen drift» in particular) of the ionosphere inhomogeneities is introduced, time series of the field can then be produced.

To solve the problem of propagation inside the ionosphere layer, the complex phase method was extended to the most general case of a 3D background medium (Gogin et al., 2001). According to this extension the first order complex phase is represented as

$$
\begin{aligned}
& \psi_{1}\left(s_{0}, 0,0\right)=-\frac{k^{2}}{4 \pi} \iiint d s d q_{1} d q_{2} \frac{\varepsilon\left(s, q_{1}, q_{2}\right)}{n_{0}(s)} h_{s}\left(s, q_{1}, q_{2}\right)\left[\operatorname{det} \hat{B}^{+}(s)\right]^{1 / 2} . \\
& \cdot \exp \left\{\frac{i k}{2}\left[\left(b_{11}+b_{11}^{g}\right) q_{1}^{2}+\left(b_{22}+b_{22}^{g}\right) q_{2}^{2}+2\left(b_{12}+b_{12}^{g}\right) q_{1} q_{2}\right]\right\} .
\end{aligned}
$$

Here $n_{0}(s)=n(s, 0,0)=\left[\varepsilon_{0}(s, 0,0)\right]^{1 / 2} \cdot \hat{B}^{+}=\hat{B}+\hat{B}^{-}$. Matrices $\hat{B}$ and $\hat{B}^{g}$ with the elements $b_{i k}, i, k=1,2$ and $b_{i k}^{g}, i, k=1,2$ respectively, satisfy matrix Riccati equations

$$
\begin{gathered}
n_{0} \frac{\partial \hat{B}}{\partial s}+\hat{B} \cdot \hat{B}=\hat{C} \\
-n_{0} \frac{\partial \hat{B}}{\partial s}+\hat{B}^{g} \cdot \hat{B}^{g}=\hat{C} .
\end{gathered}
$$

Equations ((15.8) to (15.10)) are written in a ray-centered co-ordinate system with variable along the reference ray, where the reference ray is a ray linking a transmitter and receiver in the undisturbed ionosphere and transversal variables $q_{1}$ and $q_{2}$ lie in the plane perpendicular to a reference ray at each point. Equations ((15.3) and (15.4)) are solved numerically along with the appropriate ray-tracing code describing the 3D background ionosphere.

Procedure of generating random series of a field based on the complex phase method is described in Gherm et al. (2002a) for the plane-stratified background medium. It is the same in the general case of the 3D background medium.

Briefly, to generate random realizations of the field at the bottom of the ionosphere (i.e. to generate an appropriate random screen below the ionosphere) the spatial spectra of the correlation functions of the log-amplitude $\chi_{1}: B_{\chi}\left(\omega ; q_{1}, q_{2}\right)=\left\langle\chi_{1}\left(\omega ; q_{1}\right) \chi_{1}\left(\omega ; q_{2}\right)\right\rangle$ phase $S_{1}: B_{S}\left(\omega ; q_{1}, q_{2}\right)=\left\langle S_{1}\left(\omega ; q_{1}\right)\right.$. $\left.\cdot S_{1}\left(\omega ; q_{2}\right)\right\rangle$ of the random field $\left(\psi_{1}=\chi_{1}+i S_{1}\right)$ and their cross-correlation function $B \chi_{S}\left(\omega ; q_{1}, q_{2}\right)=$ $=\left\langle\chi_{1}\left(\omega ; q_{1}\right) S_{1}\left(\omega ; q_{2}\right)\right\rangle$ are needed, as well as the Probability Density Functions (PDF) for fluctuations of $\chi_{1}$ and $S_{1}$. Appropriate representations for these functions are available for the general case of a 3D background ionosphere, once representation (15.2) has been derived.

As far as the PDFs for $\chi_{1}$ and $S_{1}$ are concerned, both these quantities, according to eq. (15.2) are represented as a sum of a large number of the statistically independent random contributions (the integral in (15.2) is over a large number of random inhomogeneities). According to the central limit theorem, this means that both the random functions $\chi_{1}$ and $S_{1}$ are normally (gaussian) distributed.

Some results presented below, which illustrate the functioning of the method, have been calculated for the following model case: NeQuick model profile of the background ionosphere for low-latitude ionosphere with TEC $=69$ TECU; the spectral index of the inverse power spatial spectrum of the electron density fluctuation $p=3.7$; the variance of the relative electron fluctuations is $\sigma_{N}^{2}=10^{-2}$; the cross-field outer scale of $10 \mathrm{~km}$, aspect ratio $a=5$, the elevation angle of the path of propagation of $45^{\circ}$ and its azimuth of $45^{\circ}$; the effective velocity of the horizontal frozen drift of $300 \mathrm{~m} / \mathrm{s}$; the transmission frequency of $500 \mathrm{MHz}$.

Figure 15.3 presents 2D realizations of the random distributions of phase (left panel) and log-amplitude (right panel) for the level of the Earth's surface. 

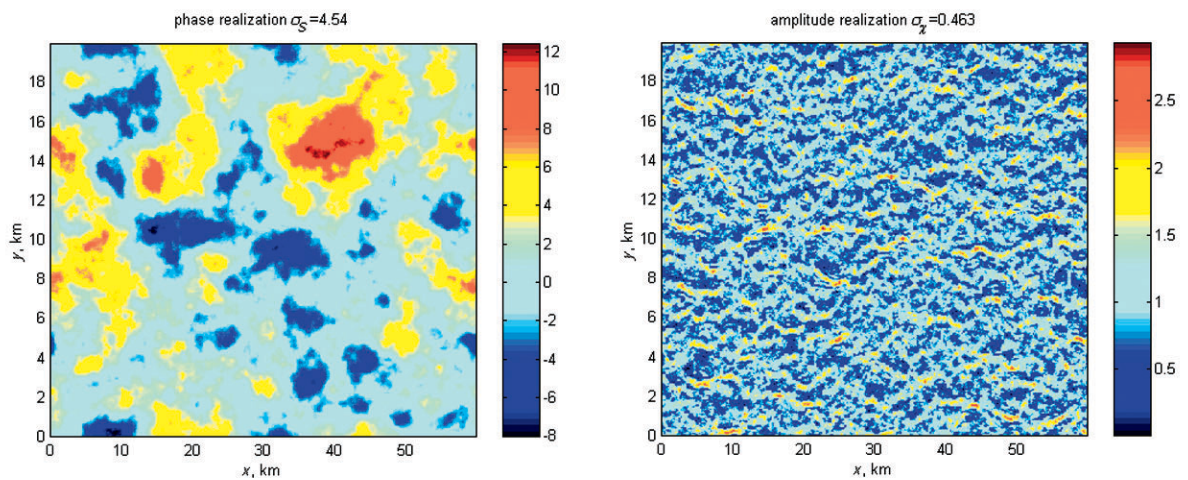

Fig. 15.3. Phase and amplitude realizations of the medium.
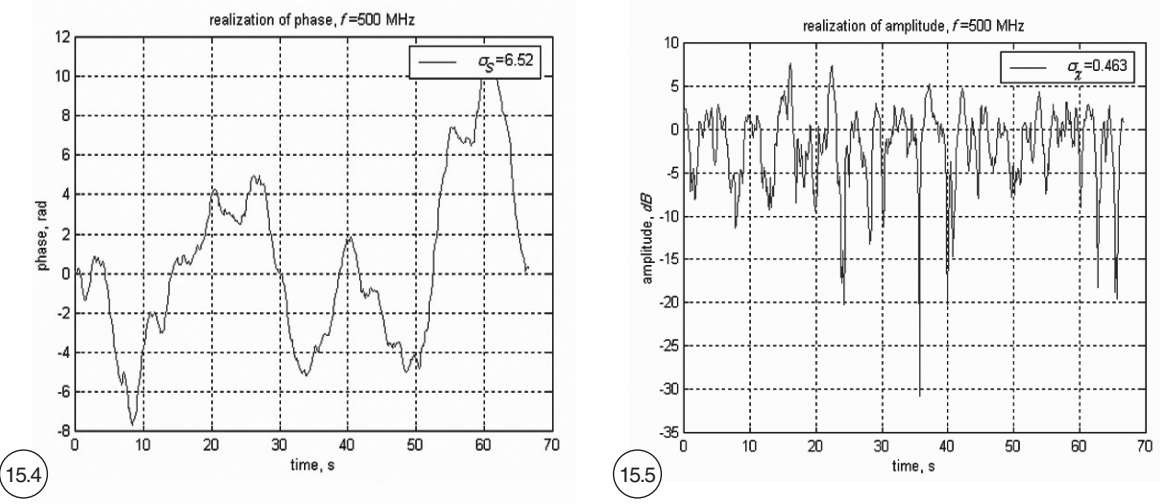

Fig. 15.4. Phase of transmitted signal.

Fig. 15.5. Intensity of transmitted signal.

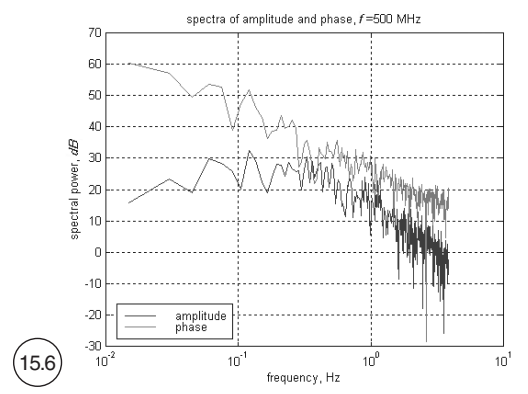

(15.7)

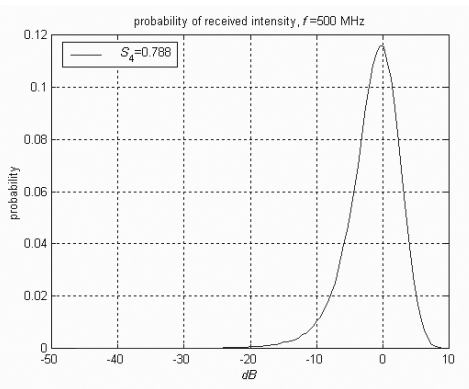

Fig. 15.6. Phase and spectrum of received signal.

Fig. 15.7. Probability of received intensity. 
When the hypothesis of the frozen drift is accepted, distributions represented in fig. 15.3 are transformed to random time series for the phase (fig. 15.4) and amplitude of the field (fig. 15.5).

Figure 15.6 present the frequency spectra of the phase and log-amplitude fluctuations of the field simulated for the regime of strong scintillation $S_{4}=0.789$. From the point of view of the forms of the log-amplitude and phase spectra, the regime of strong scintillation is characterized by the fact that neither curves merge at the high-frequency tail. This distinguishes the case of strong scintillation from the case of weak scintillation for which both the curves of the phase and the log-amplitude spectra merge at high frequencies.

Additionally, in the case of strong scintillation the probability density function for the amplitude fluctuations is of asymmetric form, as is seen in fig. 15.7.

A similar technique also permits investigation of the effects of scintillation on signal coherency over spaced paths of propagation (Gherm et al., 2001).

\subsection{SCINTILlations AT RECEIVER LEVEL}

\subsubsection{GPS receiver architecture}

A GPS receiver is a spread spectrum receiver, requiring several essential parts for acquisition, tracking and extracting useful information from the incoming satellite signal. It can be broadly divided into three sections: the RF Front-end (RFF), Digital Signal Processing (DSP) and the Navigation Data Processing (NDP). The RFF and the DSP sections generally consist of various hardware modules, whereas the NDP section is implemented using software. Figure 15.8 shows a simple block diagram of a typical single frequency GPS receiver with major interfaces and input/output signals of the essential blocks.

The DSP performs the acquisition and tracking of the GPS signal. Traditional signal demodulation such as those used for FM or AM cannot be used for spread spectrum signals such as GPS because the signal level is below the noise level. Instead, the signal must be coherently integrated over time so that the noise is averaged out, thereby raising the signal above the noise floor.

Any GPS receiver locking onto a GPS satellite signal has to do a two dimensional search for the signal. The first dimension is time. The GPS signal structure for each satellite consists of a 1023 bit long Pseudo-Random Number (PRN) sequence sent at a rate of $1.023 \mathrm{Mbits} / \mathrm{s}$, i.e. the code repeats every millisecond. To acquire in this dimension, the receiver needs to set an internal clock to the correct one of the 1023 possible time slots by trying all possible values. Once the correct delay is found, it is tracked with a Delay Lock Loop (DLL).

The second dimension is frequency. The receiver must correct for inaccuracies in the apparent doppler frequency. Once the carrier frequency is evaluated, it is tracked with a Phase Lock Loop (PLL). Figure 15.9 shows an extremely simplified PLL/DLL architecture. A more precise description of the GPS signal processing can be found in Ward (1996).

\subsubsection{Phase noise at receiver level}

When the receiver is unable to track the carrier phase, the signal is lost. Loss of lock is directly related with PLL cycle slips. To evaluate the occurrence of cycle slips, the tracking error variance at the output of the PLL as to be considered. This variance is expressed as a sum of three terms (Conker et al., 2003)

$$
\sigma_{\phi}^{2}=\sigma_{\phi s}^{2}+\sigma_{\phi T}^{2}+\sigma_{\phi, o s c}^{2}
$$




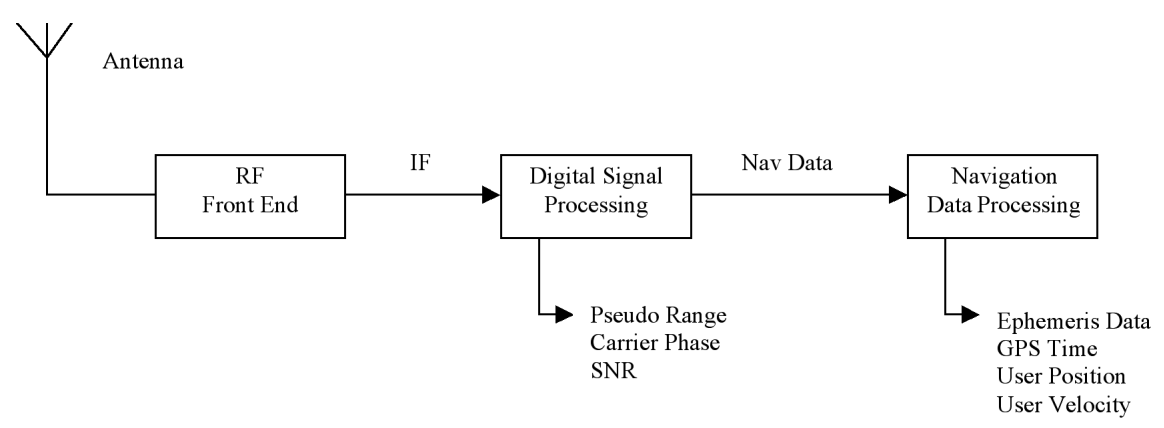

Fig. 15.8. Block diagram of a generic GPS receiver.

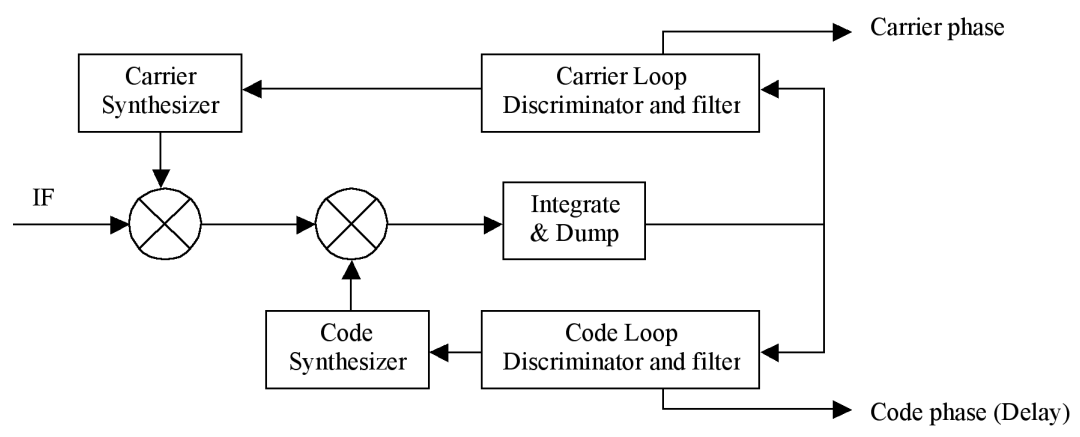

Fig. 15.9. Simplified GPS Digital Receiver Channel.

where $\sigma_{\phi s}$ is the phase scintillation variance, $\sigma_{\phi T}$ is the thermal noise variance and $\sigma_{\phi, \text { osc }}$ is the receiver oscillator noise (0.122 rad) (Conker et al., 2003).

The phase variance scintillation at the output of the PLL is given by

$$
\sigma_{\phi s}^{2}=\int_{-\infty}^{\infty}|1-H(f)|^{2} S_{\phi}(f) d f
$$

where $S_{\phi}(f)$ is the PSD of phase scintillation (Conker et al., 2003).

Figure 15.10 shows a phase scintillation spectrum obtained with GISM. $|1-H(f)|^{2}$ is the closed loop transfer function of the PLL and depends on $k$, the loop order, and fn, the loop natural frequency. Its expression is given by (15.13). Typical values are $k=3$ and $f_{n}=1.91 \mathrm{~Hz}$

$$
|1-H(f)|^{2}=\frac{f^{2 k}}{f^{2 k}+f_{n}^{2 k}} .
$$

When there is no scintillation, the standard thermal noise tracking error for the PLL is

$$
\sigma_{\phi T}^{2}=\frac{B_{n}}{\left(c / n_{0}\right)}\left[1+\frac{1}{2 \eta\left(c / n_{0}\right)}\right]
$$


where $c / n_{0}$ is the Signal to Noise Ratio (SNR), $B_{n}$ is the receiver bandwidth, and $\eta$ is the predetection time. For airborne GPS receiver, $B_{n}=10 \mathrm{~Hz}$ and $\eta=10 \mathrm{~ms}$. Amplitude scintillation alters the SNR and increases the thermal noise tracking error. According to (Conker et al., 2003), in the presence of scintillation characterized by $S 4$ index, thermal noise tracking error is given by

$$
\sigma_{\phi T}^{2}=\frac{B_{n}\left[1+\frac{1}{2 \eta\left(c / n_{0}\right)\left(1-2 S 4^{2}\right)}\right]}{\left(c / n_{0}\right)\left(1-2 S 4^{2}\right)} .
$$

Equation (15.15) needs the evaluation of the SNR.

The GPS link budget can be expressed in $\mathrm{dB}$ as follows:

$$
C / N_{0}=P_{0}+G t+G r-\text { Propagation losses }- \text { Insertion Losses }-N_{0}
$$

where $P_{0}$ is the emitted power, $G t$ and $G r$ are respectively the emitter and the receiver antenna gain, and $N_{0}$ is the receiver noise density. Therefore, the SNR appears to depend on the elevation angle as shown in figure 15.11.

Equations ((15.15) and (15.12)) can be used with (15.11) to compute the PLL tracking error variance. Figure 15.12 is a comparison of this variance versus $C / N_{0}$ for $S 4=0.7$ and $S 4=0.5$. Loss of lock is highly probable for values above the $15^{\circ}$ threshold. Therefore a receiver is able to tolerate scintillation if the $C / N_{0}$ is above a minimum value. This minimum is $26 \mathrm{~dB}$ for $S 4=0.5$ and $32 \mathrm{~dB}$ for $S 4=0.7$.

\subsection{Loss of Lock Probability}

Thermal noise appears to be the essential contribution to PLL tracking error. It is the unique $S 4$ dependent term in (15.11) and the influence of $S 4$ is obvious in fig. 15.12. A study of amplitude scin-
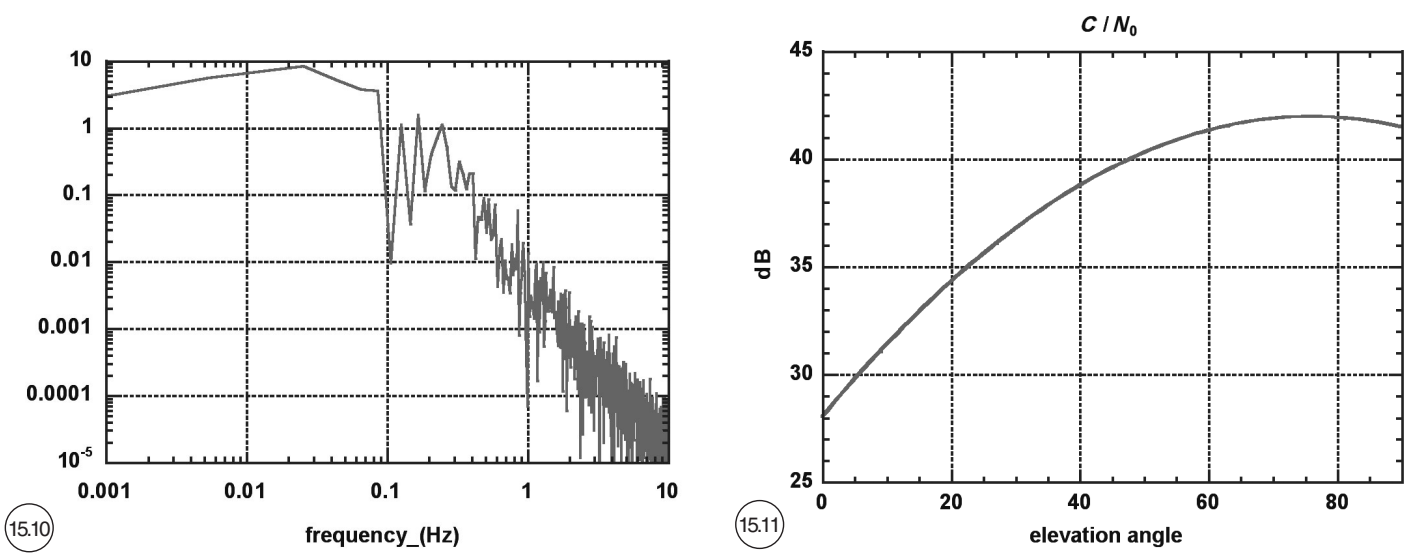

Fig. 15.10. PSD of phase scintillation computed with GISM.

Fig. 15.11. $C / N_{0}$ versus elevation angle without scintillation for a GPS link. 

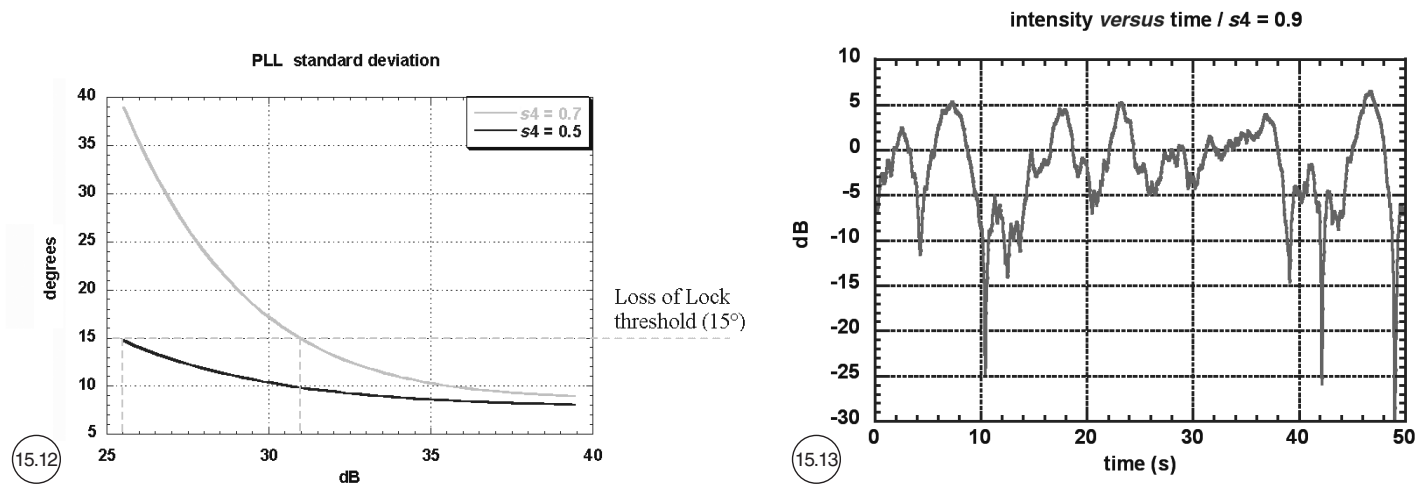

Fig. 15.12. PLL standard deviation versus $C / N_{0}$.

Fig. 15.13. Scintillation intensity versus time computed with GISM.

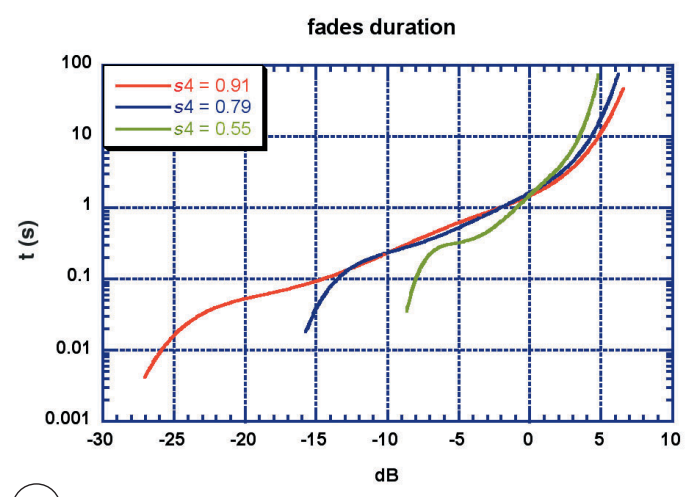

(15.14)

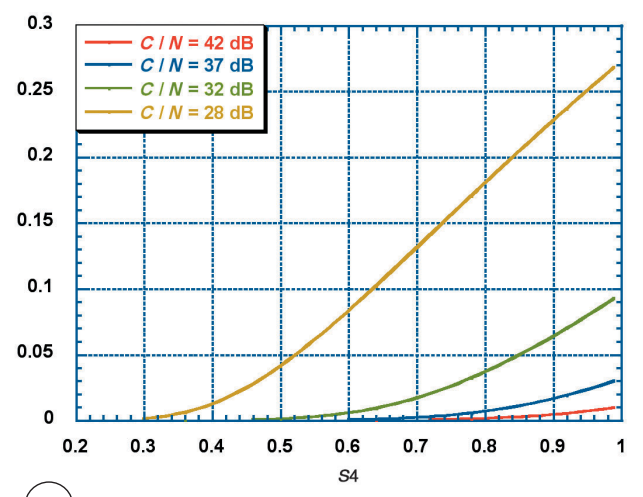

(15.15)

Fig. 15.14. Fades duration versus fade depth.

Fig. 15.15. Probability of loss of lock versus $S 4$ for 4 values of the SNR.

tillations is detailed in Conker et al. (2003) and leads to (15.15). We will present here another approach of amplitude scintillation effects on thermal noise.

Figure 15.13 shows a typical signal amplitude under severe scintillation conditions $(S 4=0.9)$. The corresponding fade duration (fig. 15.14) always exceeds the pre integration duration time. As a consequence it corresponds to a degradation of the SNR at receiver level

$$
C / N=C / N_{0}+I S \quad(\text { in } \mathrm{dB})
$$

or, with the fractional form

$$
c / n=c / n_{0} * I s
$$


where $I s$ is the scintillation intensity. Its mean value is 1 and it has a Nakagami distribution characterized by $S 4$.

Equation (15.10) is modified to take the fading into account:

$$
\sigma_{\phi T}^{2}=\frac{B_{n}}{\left(c / n_{0}\right) I s}\left[1+\frac{1}{2 \eta\left(c / n_{0}\right) I s}\right] .
$$

This relation expresses thermal noise as a decreasing function of scintillation intensity. As a result, if $\sigma_{\phi T}$ is above the $15^{\circ}$ threshold then $I s$ is below a value computed using (15.19). As Is distribution is known for a given $S 4$, the probability of occurrence of «Is $<$ threshold» can be evaluated. The result is the probability of Loss of Lock. Figure 15.15 presents this probability versus $S 4$ at given values of the SNR. It can be noticed that links with high SNR are quite robust. On the contrary, links with low values of SNR are likely to be lost.

GISM has an integrated GPS satellite trajectory generator. It has been used use to simulate a whole day (24th September 2001) over Naha (Japan, latitude $=26^{\circ}$ geographic, $15^{\circ}$ magnetic). All visible satellites were used to compute an average probability of loss of lock. The result is $0.21 \%$. In other words, each satellite was $0.21 \%$ of the time locked out during that day.

\subsection{Positioning Errors}

In most cases, scintillations do not affect all visible satellites. If there are more than four satellites then a standard receiver should be able to provide navigation information. However, the number of satellites and their positions affect the positioning precision. The Dilution Of Precision (DOP) is usually used to quantify this precision. The DOP is related to the geometrical distribution of the visible constellation. The scheme in fig. 15.12 shows how the DOP is related to the satellites positions. The DOP is used to derive the positioning error $\left(\sigma_{p}\right)$ from the User Equivalent Range Error (UERE)

$$
\sigma p=\mathrm{DOP} * \mathrm{UERE} \text {. }
$$

GISM was used to compute all scintillation parameters for each GPS satellite visible from Naha (Japan). The tracking error was derived from these parameters and from typical receiver characteris-

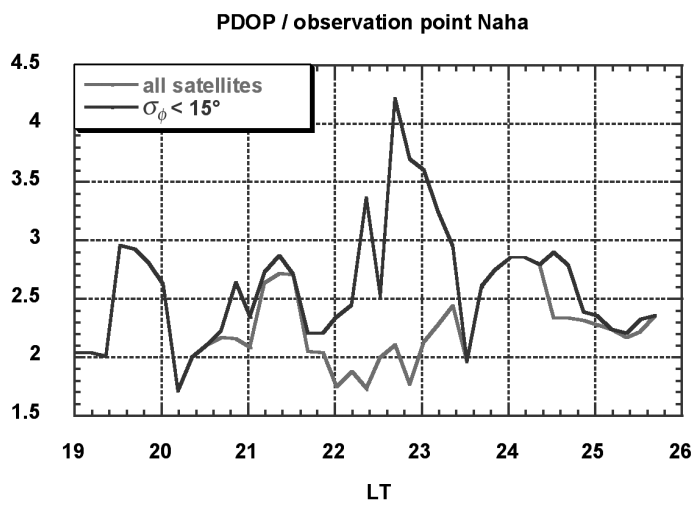

Fig. 15.16. DOP at Naha under scintillation conditions computed with GISM. 

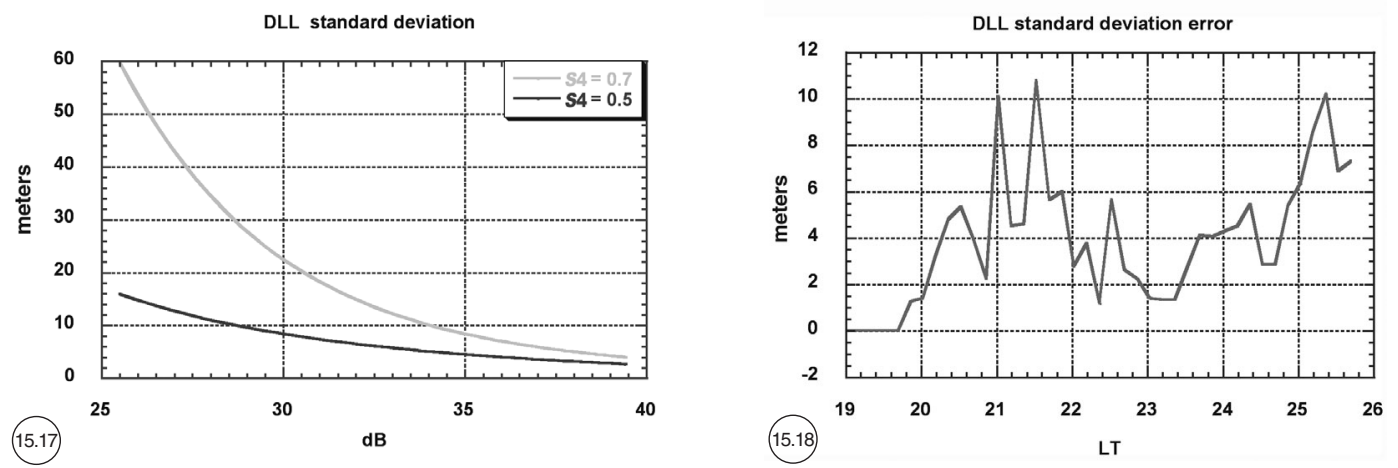

Fig. 15.17. DLL tracking error versus $C / N_{0}$.

Fig. 15.18. Positioning error at Naha (Japan) under scintillation conditions, computed with GISM.

tics. Satellites with tracking error above the $15^{\circ}$ threshold were ignored for the DOP evaluation. Figure 15.16 presents the resulting DOP, compared with the DOP of the unaffected constellation. In worst cases, the DOP during scintillation can be twice as high as under normal conditions.

Even if the signal emitted from a GPS satellite is not lost, it can alter the position precision. One of the DLL functions is the measurement of the delay between the code carried by the GPS signal and the receiver internal clock. This delay is an estimation of the time needed by the GPS signal to reach the receiver. The receiver is then able to compute the distance of the satellite. Errors in this estimation are collected in the UERE. To take into account the scintillations, we have to consider the DLL tracking errors.

The DLL can be studied like the PLL to evaluate its tracking error variance in degrees. The UERE due to scintillations can then be deduced with a product with the chip length (equal to $293 \mathrm{~m}$ for $L 1$ ). The results are shown in fig. 15.17. These results seem to show high degradation of the UERE. Satellites with high DLL tracking errors also have high PLL tracking errors and therefore they might be considered as lost and don't contribute to the UERE.

The combination of both effects is presented in fig. 15.18. Satellites with PLL tracking errors above $15^{\circ}$ were considered invisible for the DOP calculation. All other links with visible satellites were used to compute a mean UERE contribution due to scintillation.

\subsection{Measurement TEChNiQues AND DATA PROCESSING ALgORITHMS}

\subsubsection{General considerations}

During recent observations made by means of GPS scintillation monitors, at high latitudes high phase scintillations have usually been measured for low intensity scintillations (Doherty et al., 2000; Pi et al., 2001). During a Wideband experiment, cases of high phase scintillation against low intensity scintillation were seldom found (Fremouw et al., 1978).

Very recently, it has been shown that an erroneous data detrending can be responsible for distortions in the information gained from experimental data measured by GPS scintillation monitors. Data detrending, when the separation of deterministic and stochastic components is done using a fixed 
cut-off frequency and not appropriate to the actual geophysical conditions occurring during measurements, can lead to a misleading data interpretation (Forte et al., 2002). The comparison of GPS observations with Low Earth Orbit (LEO) satellite beacon data is difficult. The GPS to ground rays scan through the irregularities in varying directions and with low velocity.

For the Wideband satellite experiment a detrend filter with a fixed cut-off frequency of $0.1 \mathrm{~Hz}$ was carefully chosen. This was a correct choice for the case of an orbiting satellite with a scan velocity of about $3 \mathrm{~km} / \mathrm{s}$ in the $F$-layer, as for the Wideband satellite. In the case of scintillation experiments with GPS satellites (having a much lower scan velocity of about $80 \mathrm{~m} / \mathrm{s}$ ), use of the same $0.1 \mathrm{~Hz}$ detrend filter may not be appropriate. This can lead to distortions of intensity and phase scintillation data. The distortions include the possibility of obtaining phase without amplitude scintillation, as reported in literature (Doherty et al., 2000).

Scintillation results obtained at high latitudes during two measurement campaigns in Finland and Norway are presented below.

\subsubsection{Results obtained in Finland}

An experiment for measuring data detrending effects on ionosphere scintillation information pointed out from GPS signals was set up at auroral latitudes. The experiment is run at the Sodankyla Geophysical Observatory (University of Oulu). A GPS receiver with a sufficiently high sampling rate has been used to measure the carrier phase and signal-to-noise ratios at $L 1$. The use of GPS signal scintillation allows measurements assessing possible mechanisms responsible for high phase scintillation with low amplitude scintillation, as observed by means of GPS scintillation monitors at high latitudes.

\subsubsection{Description of the experiment}

GPS signals are tracked and analyzed by means of a JNS100 Javad GPS receiver. The OEM board has 50 channels, all in view: L1 GPS, INMARSAT, WAAS/EGNOS and GLONASS. It is characterized by low signal power tracking (down to $30 \mathrm{~dB} * \mathrm{~Hz}$ ), fast acquisition and re-acquisition, up to $30 \mathrm{~g}$ 's of dynamic, advanced multipath mitigation, $10 \mathrm{~cm}$ code phase and $0.1 \mathrm{~mm}$ carrier phase precision in differential modes. It is connected to a normal PC by four high speed (115.2 Kbps) standard RS232 serial ports. It allows to record position and raw data at sampling rates up to $100 \mathrm{~Hz}$.

This experiment was installed at the Sodankyla Geophysical Observatory (University of Oulu) and has been running since June 2003. The antenna was placed on the top of a $10 \mathrm{~m}$ tower, to avoid as far as possible multipaths coming from the ground or from obstacles on the ground. An elevation mask of $10^{\circ}$ is applied to the measurements. Carrier phase and signal-to-noise ratios on $L 1$ are measured at $20 \mathrm{~Hz}$ sampling rate. For the particular observation site this was a good tradeoff between storage capability and spectral resolution of the phenomena under investigation.

The raw data are recorded in two different formats (one standard format provided by Javad and the other specially designed for this experiment). The data are recorded continuously from 16 LT until 08 LT. The measured data are first recorded and then converted from binary to ASCII format by means of a specially designed software. Then, analysis of the data and computation of scintillation indices, for both amplitude and phase scintillation, takes place. The boundary conditions characterizing the detrending operations are carefully evaluated and calibrated to the geophysical conditions. Phase and intensity power spectral densities are estimated to characterize the signal fluctuations originated by small-scale irregularities. The detrending algorithm is based on a FIR windowed filter allowing the separation of low and high frequency signal fluctuations. 


\subsubsection{Example of output data}

One example of detrended carrier phase and intensity is shown on fig. 15.19. This example shows the high frequency signal residuals, after separating ionosphere small-scale effects from other undesired effects, as multipath, satellite motion, clock biases, etc. From these high frequency signal residuals, power spectral densities can be estimated (fig. 15.20). From the intensity power spectral density the Fresnel frequency can be estimated around $1 \mathrm{~Hz}$. This estimate confirms the Fresnel frequency shift towards high values at high latitudes. This was theoretically suggested as a concurrent factor in the explanation of high phase scintillation against low intensity scintillation measured at high latitudes (Forte et al., 2002).

Scintillation indices corresponding to the raw data presented in fig. 15.19 are shown in fig. 15.21. Both $\sigma_{\phi}$ and $S 4$ are shown for this particular case, corresponding to different filtering conditions. Curves with symbols «+» and «*» indicate $S 4$ and $\sigma_{\phi}$ corresponding to a cut-off detrending frequen-
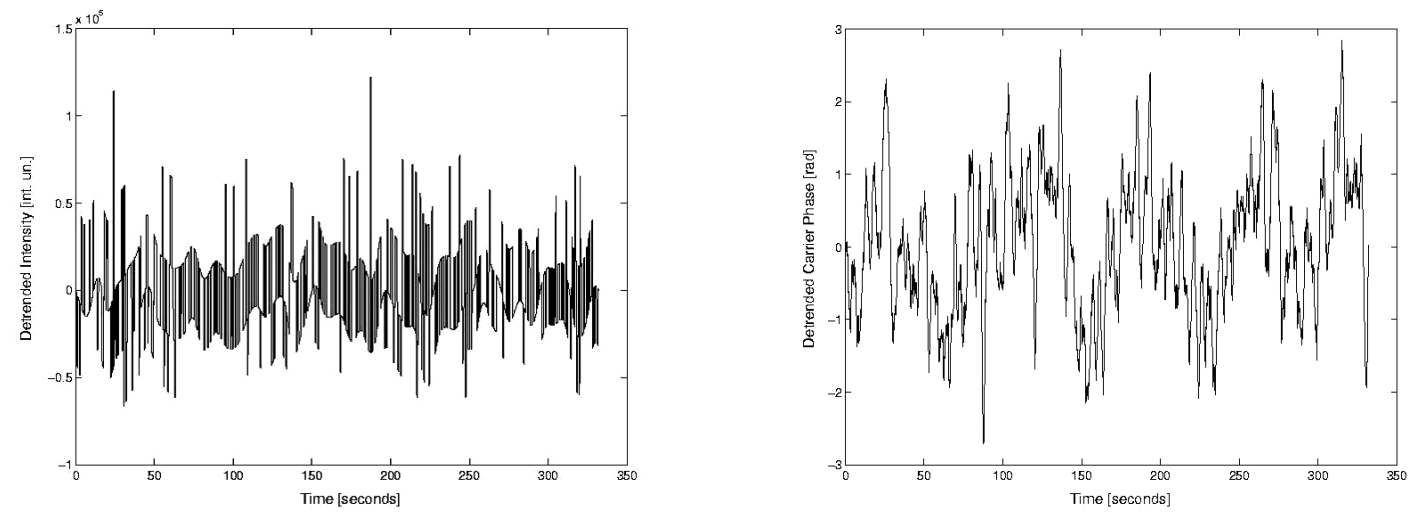

Fig. 15.19. High frequency residual of received intensity and phase for PRN01 on 24.04.03 at 20 UT.
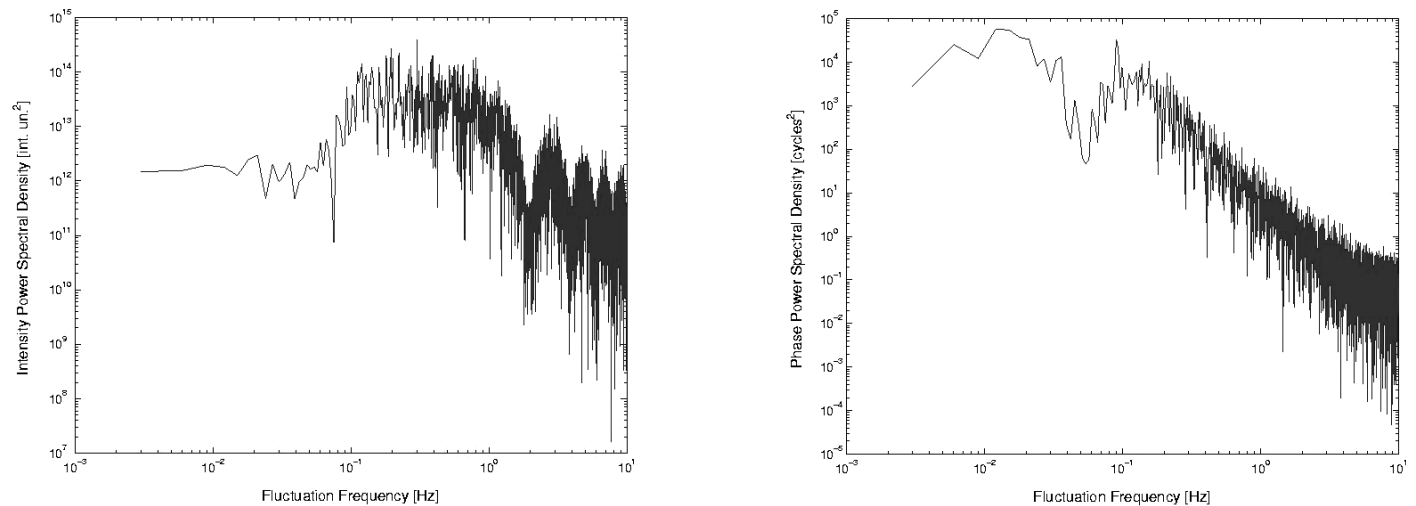

Fig. 15.20. Intensity and phase PSD after detrending. 


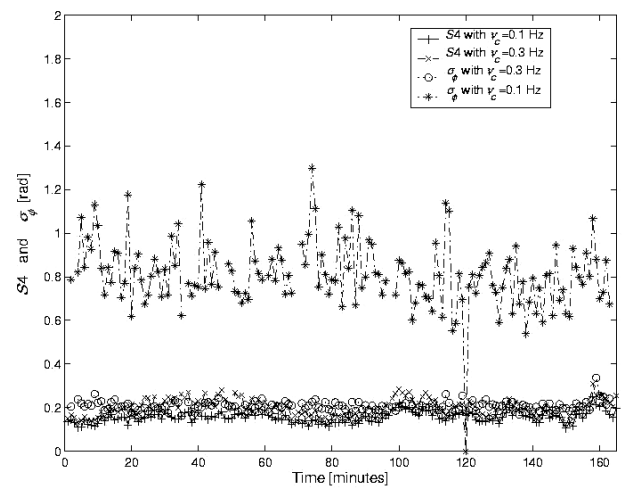

Fig. 15.21. Intensity and phase scintillation indices varying the filtering parameters (raw data of fig. 15.19).

PRN 9

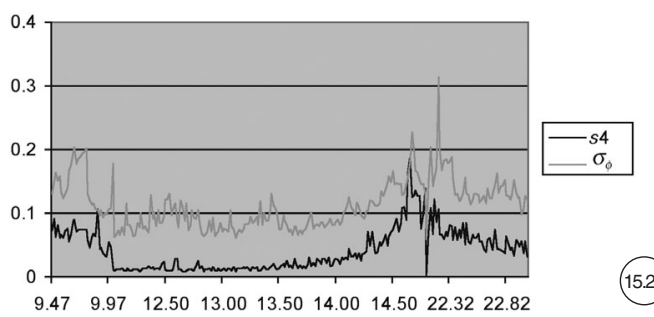

Average values for the second week of January in Tromso

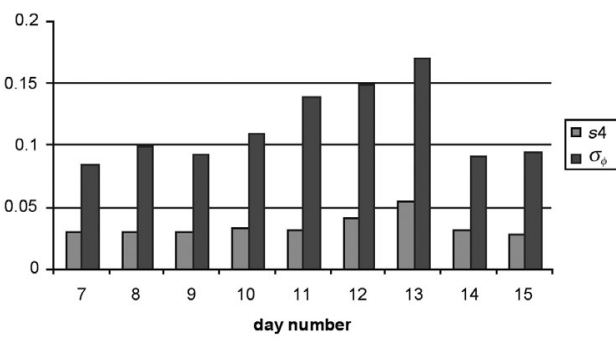

Fig. 15.22. Scintillations recorded in Norway.

Fig. 15.23. Phase and intensity scintillations levels.

cy of $0.1 \mathrm{~Hz}$, while curves with symbols «x» and «O» indicate $S 4$ and $\sigma_{\phi}$ corresponding to a cut-off detrending frequency of $0.3 \mathrm{~Hz}$ (Forte and Radicella, 2003). As can be seen, $S 4$ is slightly affected by this change in the detrending cut-off frequency, maintaining approximately the same values and behavior in the two cases («+» and «x» symbols). The phase scintillation index $\sigma_{\phi}$, on the contrary, is critically affected by such a change: while curve «*» contains not only contributions from diffractive scattering but also from refractive scattering, the curve «O» contains just diffractive terms, as happens for the intensity scintillation indices $S 4$. As mentioned before, incorrect setting of this parameter may strongly affect the results of phase scintillation.

\subsubsection{Results obtained in Norway}

DLR (Neustrelitz, Ge) has installed one experimental set up in Tromsø, Norway. A Javad receiver is used. An algorithm has been developed and implemented to obtain $S 4$ and $\sigma_{\phi}$ from the $50 \mathrm{~Hz}$ 
Rinex files. The calculation of $S 4$ is performed from the CA code. Small fluctuations on the satellite motion or trajectory do not affect its value significantly.

On the contrary, due to the high value of the carrier frequency $(L 1)$, small fluctuations of the satellite attitude on the trajectory significantly affect the calculation of $\sigma_{\phi}$. A high order filter has been implemented to retrieve the effects due to the satellite attitude and motion. Since these effects are slower than the ionosphere scintillation, the carrier phase can be detrended before standard deviation computation. A digital high-pass Butterworth filter has been implemented to detrend the phase.

A typical result is presented below (fig. 15.22).

The phase scintillation includes implicitly the phase receiver noise as indicated in Section 15.5. As general observations, it can be mentioned that both phase and intensity scintillations exhibit low values and that they are strongly correlated (fig. 15.23).

\subsection{COMPARISONS BETWEEN RESULTS OBTAINED WITH SCINTILLATION MODELS AND MEASUREMENTS}

Several modeling efforts have been made up to now to describe the physical mechanism giving rise to scintillation as well as the statistical distribution of ionosphere electron density irregularities. The most important goal is the forecast of scintillation activity. This is required in the evaluation of ionosphere scintillation impact on important applications of radio wave propagation, such as satellite based navigation and positioning.

\subsubsection{WBMOD}

The WBMOD model describes a worldwide climatology of the ionosphere plasma density irregularities which can produce scintillation of radio waves traversing the ionosphere. WBMOD is a climatological model for ionosphere electron density irregularities coupled with a propagation model able to describe the effects of ionosphere plasma irregularities on transionosphere radio signals (Fremouw et al., 1978). The parameters of ionosphere irregularities are modeled on the basis of experimental data, while the propagation model is essentially a phase screen model. The model provides the intensity scintillation index $S 4$ and the phase scintillation index $\sigma_{\phi}$, computed by means of the propagation model under the pre-specified geophysical conditions. The model can compute the percentage of time that those indices would exceed a specified threshold level for given geophysical conditions. It is also able to compute the SI scintillation index, which has engineering applications.

\subsubsection{GISM}

Global Ionosphere Scintillation Model (GISM) provides the statistical characteristics of the transmitted signals, in particular scintillation indices, fade duration, and cumulative probability of the signal, allowing the determination of the margins to be included in a budget link. Maps of scintillation indices can be easily obtained. The model is composed of two models. The first model provides the mean errors and is based on a solution of the ray equation for background propagation plasma. The refractive index is calculated from an accurate value of the electron density inside the medium, which is provided by the NeQuick model (Di Giovanni and Radicella, 1990). The second model is based on the multiple phase screen technique and provides second order propagation effects, namely radio wave scintillation. Inputs to the model consist in the geophysical parameters, inhomogeneity data, and the operating data (e.g., carrier frequency, link geometry, local time, etc.). The inhomogeneity data are essentially deduced from measurements. Intensity and phase scintillation indices are provided as outputs (Béniguel, 2002). 


\subsubsection{Measurements recorded at Tucuman (Argentina)}

The data set used for this study was collected at the Institute of Physics of the National University of Tucuman (Argentina), by means of a scintillation monitor developed and provided by Cornell University (Forte et al., 2002). S4 scintillation indices averaged over one minute periods are computed. Data were collected over 11 months; the measurements time interval is of $12 \mathrm{~h}$ during nighttime.

The ionosphere over the Tucuman sky is assumed to be approximated by a thin shell at $350 \mathrm{~km}$ of altitude. Such modeled ionosphere is then interfaced with a grid subdivided into $5^{\circ} \times 5^{\circ}$ surface square boxes.

The use of an ionosphere grid at $350 \mathrm{~km}$ is suitable to subdivide spatially and temporally both experimental and modeled information. An ionosphere grid of $5^{\circ} \times 5^{\circ}$ surface square boxes is adopted. In this way a clear geographical distribution of the information can be displayed. This method, repeated for several hours, gives at the end a full spatial-temporal view of the observed and modeled phenomena, helping in evaluating not only a careful comparison between models and experimental data, but also the impact of ionosphere scintillation on GPS positioning and navigation operations.
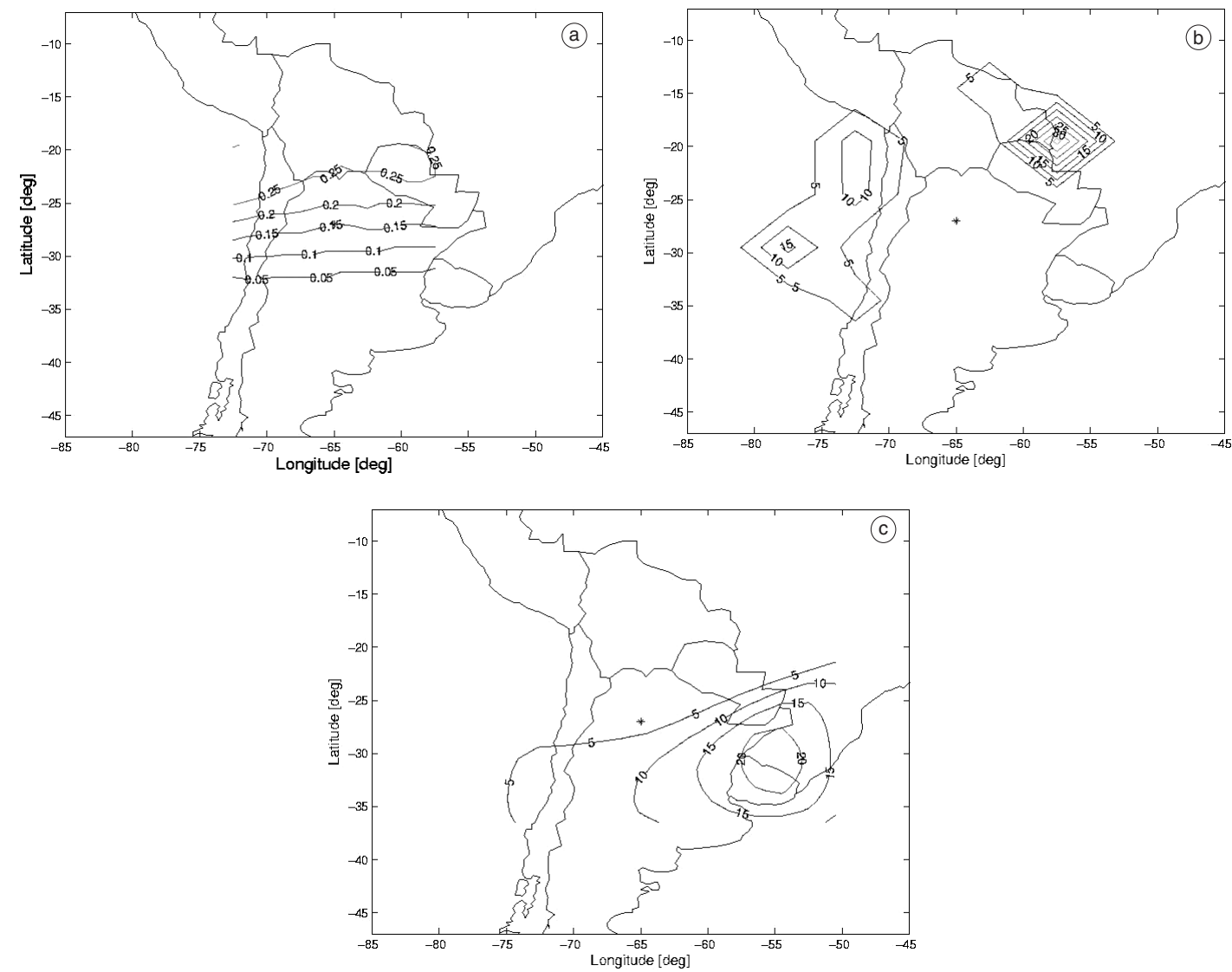

Fig. 15.24. Percentage occurrence of $S 4>0.5$ obtained from measurements (b) and calculated by GISM (a) and WBMOD (b), September 1998 at 20 UT. 
The reason for such an approach is to test the use of scintillation models for their application to operational systems like Space Based Augmentation Systems (SBAS). SBAS are GPS augmentation systems and will be used essentially in an air navigation precise approach. Three systems are planned: a) The US Wide Area Augmentation System (WAAS); b) the European Geostationary Navigation Overlay System (EGNOS); c) the Japanese MTSAT (Multifunctional Transport Satellite) satellite based augmentation system (MSAS). Basic GPS service cannot meet the accuracy, availability and integrity required by air navigation precise approach. SBAS are safety critical navigation systems that should provide a quality of positioning information never available to the aviation community before. It should improve accuracy, integrity and availability of the basic GPS service.

SBAS are based on a network of Ranging and Integrity Monitoring Stations (RIMS). Signals from GPS satellites are received by RIMS which determine the actual ionosphere error for a particular area. The corresponding correction to be applied to a single frequency positioning is computed and broadcast to a user inside a given area via a geostationary satellite. The analysis presented hereafter has been carried out in the framework of EGNOS project and its goal is to estimate the actual effects of ionosphere scintillation on GPS positioning and on the RIMS calculation of the ionosphere error in a given area. Thus, the exercise of comparing such experimental data with models prediction has been done in order to assess if theoretical models (at least those available) can be used in defining the impact of scintillations on those systems, instead of using actual and not widely available experimental data.

Penetration points are considered at ionosphere grid height, namely at $350 \mathrm{~km}$ of altitude. Information coming with experimental and modeled scintillation indices is analyzed in each ionosphere grid box. For each ionosphere grid box, percentages of occurrence of $S 4$ greater than given thresholds (i.e. $0.5,0.8$, and 0.9) are computed, in order to assess whether scintillation may have a critical impact on RIMS operations and, thus, on SBAS performance (Forte and Radicella, 2002) (fig. 15.24a-c).

\subsubsection{Measurements recorded at Naha (Japan)}

The comparisons with GPS links measurements were made for two days (Matsunaga, 2002; El Arini et al., 2003) in Naha (Japan), one with a high value of the SSN: 200 and the other with a
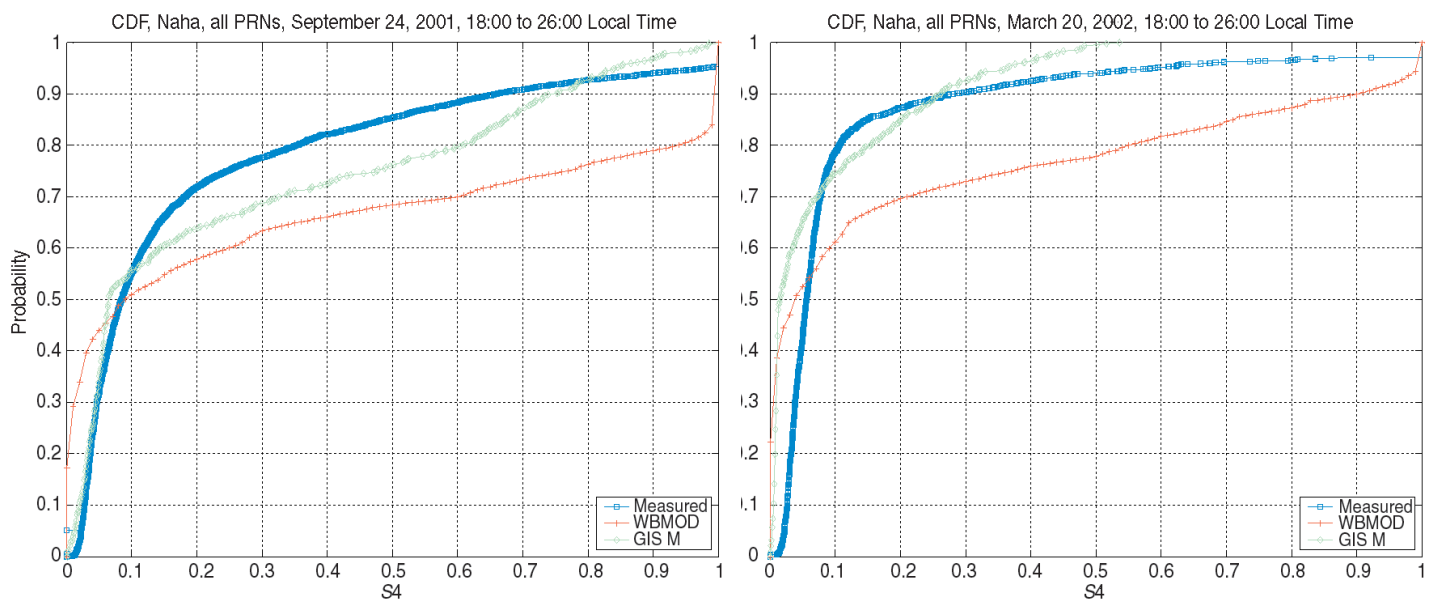

Fig. 15.25. Comparison between GISM results and measurements. Left curve $S S N=200$; right curve $S S N=85$. 

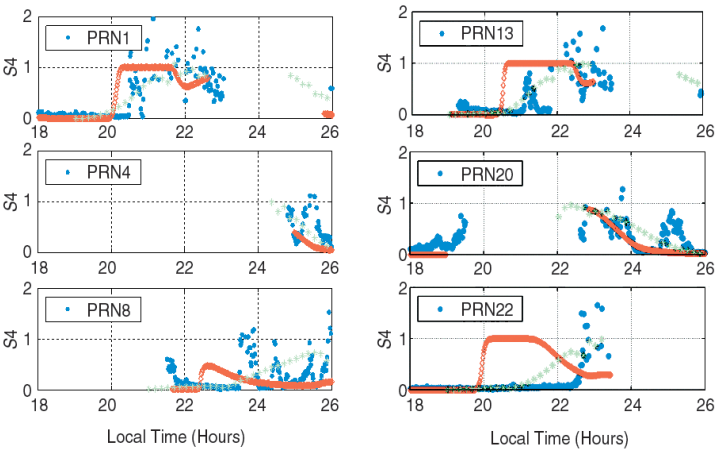

Fig. 15.26. PRN comparisons: measurements (blue); GISM (green); WBMOD (red).

low value: 85 . All the PRNs were considered. The cumulative probabilities are presented below for these two days. Measurements are in blue and GISM results are in green (figs. 15.25 and 15.26).

\subsection{Conclusions}

A review of signal scintillations has been presented. The phenomenology, essential parameters and effects on transmitted signals have been commented. Moreover the points of modeling, measurements and model - measurements comparisons have been addressed.

The receiver transfer function is a point of particular interest in the analysis. The scintillations may or may not create a loss of locks depending on the budget link which depends on the constellation parameters and on the receiver characteristics. In addition, while it is not too difficult to retrieve intensity fluctuations, the calculation of phase fluctuations is more tricky and requires a particular attention, the phase minimum value that a receiver will deliver being its phase noise value which has to be separated from the phase fluctuations due to scintillations to avoid misleading interpretations.

A database of scintillations is being constructed in Europe through measurements at high latitudes (Scandinavia) and low latitudes in Africa (Douala, Cameroun) with two objectives: to increase our knowledge regarding the physics of scintillations and develop forecasting techniques. Several projects are currently running in the frame of the space weather activity initiated by ESA, which includes the topic of scintillation forecasting. Web sites delivering such data are already active.

\section{REFERENCES}

Aarons, J. (1982): Global morphology of ionospheric scintillations, Proc. IEEE, 70 (4).

Basu, S., Su. Basu, J. La Belle, E. Kudeki, B.G. Fejer, M.C. Kelley, H.E. Whitney and A. BushBY (1986): Gigahertz scintillations and spaced receiver drift measurements during Project Condor equatorial $F$-region rocket campaign in Peru, J. Geophys. Res., 91, 5526-5538.

BÉNIGUEL, Y. (2002): Global Ionospheric Propagation Model (GIM): a propagation model for scintillations of transmitted signals, Radio Sci., May/June 2002.

Conker, R.S., M.B. El-Arini, C.J. Hegarty and T. Hsiao (2003): Modeling the effects of ionospheric scintillation on GPS/SBAS availability, Radio Sci., January/February 2003. 
Di Giovanni, G. and S.M. Radicella (1990): An analytical model of the electron density profile in the ionosphere, Adv. Space. Res., 10, p. 27.

Doherty, P., S. Delay and C. VAlladares (2000): Ionospheric scintillations effects in the equatorial and polar regions, in ION GPS Symposium, Salt Lake City (UT).

El Arini, M.B., R. Conker, Y. Béniguel and J.-P. AdAm (2003): Comparing measured $S 4$ with the calculated S4 by the WBMOD and GISM at Naha, Japan, in SBAS Meeting, September 2003, Portland (OR).

ForTe, B. and S.M. RADiCELla (2002): Problems in data treatment for ionospheric scintillation measurements, 37 (6), 1096, doi. 1029/2001RS002508.

FORTE, B. and S.M. RADICELLA (2003): Effects of ionospheric scintillation on GPS signals at auroral latitudes, in Proc. of the Atmos. Remote Sensing Conference, URSI, Matera, Italy.

ForTe, B., S.M. RADICELla and R.G. EzQUER (2002): A different approach to the analysis of GPS scintillation data, Ann. Geophysics, 45 (3/4), 551-561.

Fremouw, E.J., R.L. Leadabrand, R.C. Livingston, M.D. Cousins, C.L. Rino, B.C. FAir and R.A. LONG (1978): Early results from the DNA Wideband satellite esperiment-Complex signal analysis, Radio Sci., 13 (1), 167-187.

Gherm, V.E., N.N. ZernOv, S.M. Radicella and H.J. Strangeways (2000): Propagation model for signal fluctuations on transionospheric radiolinks, Radio Sci., 35 (5), 1221-1232.

GHerm, V.E., N.N. Zernov and H.J. StRAngeways (2001): Effect of ionospheric scintillations on signal coherency over spaced paths for transionospheric propagation, in ICAP 2001, April 2001, Manchester, U.K., Conference publication No. 480, 806-809.

Gherm, V.E., N.N. Zernov, S.M. Radicella and H.J. Strangeways (2002a): Propagation Model for Signal Fluctuations on Transionospheric Radio Links: Recent Developments, Acta Geod. Geophys., Hung., 37 (2-3), 271-280.

GHerm, V.E., N.N. Zernov, H.J. Strangeways, B. Forte and S.M. RAdicella (2002b): Some fine effects in propagation problem for transionospheric satellite radiolinks, in Proceedings 27th General Assembly of URSI, Maastricht, The Netherlands, Paper 1496, pp. 4.

Gogin, YU.A., V.E. GHERM and N.N. ZERnOv (2001): Diffraction of the wave field on weak inhomogeneities of the dielectric permittivity in a 3D smoothly inhomogeneous medium, St. Petersburg University Herald, Series 4, 2 (12), (in Russian).

MatsunAGA, K. (2002): Observation of ionospheric scintillations on GPS signals in Japan, in ION Symposium.

Pi, X., B. Boulat, A.J. Mannucci, M. Reyes and D. Stowers (2001): Ionospheric scintillations measured using GPS receivers during the current solar maximum, paper presented at the International Beacon Satellite Symposium, URSI, Boston.

WARD, P. (1996): Satellite signal acquisition and tracking, in Understanding GPS Principles and Applications, edited by E.D. KAPLAN (Artech House, Boston), 119-208. 